\title{
'THE APPLICATION TO DIRICHLET'S SERIES OF BOREL'S EXPONENTIAL METHOD OF SUMMATION
}

\author{
By G. H. HARDY.
}

[Received August 29th, 1909.--Read November 11th, 1909.]

\section{The series}

where

$$
\begin{gathered}
\sum a_{n} e^{-\lambda_{n} s}, \\
\lambda_{n+1}>\lambda_{n}, \quad \lambda_{n} \rightarrow \infty,
\end{gathered}
$$

is called a generalised Dirichlet's series.* If $\lambda_{n}=n$, it is a power series in $x=e^{-s}:$ if $\lambda_{n}=\log n$, it is an ordinary Dirichlet's series

$$
\Sigma a_{n} n^{-s} \text {. }
$$

The series (1) possesses two lines of convergence

$$
R(s)=s_{0}, \quad R(s)=\bar{s},
$$

where $s_{0} \leqslant \bar{s}$ : the series is convergent to the right of the tirst and absolutely convergent to the right of the second. Thus the series

$$
\Sigma(-1)^{n-1} n^{-s}
$$

which represents the function

$$
\left(1-2^{1-s}\right) \xi(s)
$$

is convergent for $R(s)>0$, and absolutely convergent for $R(s)>1$. On the other hand, for the series $\Sigma n^{-s}$, we have $s_{0}=\bar{s}=1$. It is evident that in the case of any ordinary Dirichlet's series $0 \leqslant \bar{s}-s_{0} \leqslant 1$. We may, of course, have $s_{0}=\bar{s}=-\infty$, the series then converging abso-

* The literature which concerns Dirichlet's series in general, considered as functions of a complex variable $s$, is rather scattered. The theory was first attacked seriously by Cahen : "Sur la fonction $\zeta(s)$ de Riemann et sur des fonctions analogues," Annales Sc. de l'École Normale Supérieure (sér. 3), t. xI, p. 75. See the dissertation of W. Schnee (Berlin, 1908), and his article in the Rendiconti del Circolo Mat. di Palermo, t. xxvir, where numerous references are given to papers by Landau and others who have contributed to the theory; a later dissertation by H. Bohr (Copenhagen, 1910); and Landau's Handbuch der Lehre von der Verteilung der Primzahlen. The general theory has been rather overwhelmed in the mass of its applications to the problem of the distribution of primes and to the general analytical theory of numbers. 
lutely all over the plane, as in the case of the series $\Sigma a^{n} n^{-s}(|a|<1)$; or, we may have $s_{0}=\bar{s}=\infty$, the series being never convergent (as in the last example when $|a|>1)$. In the general case the inequality $\bar{s}-s_{0} \leqslant 1$ need not hold: we may even have $s_{0}=-\infty, \bar{s}=\infty$, as in the case of the series

$$
\Sigma(-1)^{n-1} n^{-a}(\log n)^{-s} \quad(0<\alpha<1),
$$

which converges conditionally for all values of $s$.

When $\lambda_{12}=n, s_{0}$ and $\bar{s}$ are necessarily equal, and the function represented by the series has necessarily at least one singular point on its line of convergence. No such result holds in the general case.*

The series $\Sigma n^{-s}$ has the one singular point $s=1$, which lies on the line of convergence: and Landaut has shown that, when $a_{16}>0$, the real point on the line of convergence is always a singular point of the function represented by the series. On the other hand, the function (5) is an integral function of $s$. The line of convergence may also be a line of essential singularities, across which the function cannot be continued: Landauł has given as an example the series

$$
\Sigma 2^{-s 2^{n}} \text {. }
$$

2. These circumstances make the problem of the summation of a Dirichlet's series, even in the narrower sense, more difficult and at the same time more interesting than the corresponding problem for powerseries. In this connection some very interesting results have been stated in three recent notes in the Comptes Rendus, by MM. Bohr and Riesz.

M. Bohrs considers the application to Dirichlet's series of Cesàro's method of summation by mean values. $; \mathrm{H}$ He begins by proving the following general theorem :-

If $\Sigma a_{n}$ is summable or finite $(C k), \uparrow$ and $f_{n} \rightarrow 0$ as $n \rightarrow \infty$, and the series

$$
\Sigma\left|\Delta f_{n}\right|, \quad \Sigma_{n}\left|\Delta^{2} f_{n}\right|, \quad \ldots, \quad \Sigma_{n^{k}}\left|\Delta^{k+1} f_{n}\right|
$$

* It is easy to see, however, that $s_{0}=\bar{s}$ in all cases in which the increase of $\lambda_{n}$ is suffciently rapid to ensure the convergence of $\Sigma e^{-\lambda_{1} n^{x}}$ for all positive values of $s$.

† Math. Annalen, Bd. Lx, p. 537.

$\ddagger$ Sitzungsberichte der Akademie zu Milnchen, Bd. xxxv1, p. 191.

\$ Comptes Rendus, Jan. 11, 1909.

|| Bromwich, Infinite Series, pp. 310 et seq.

- Summable $(C k)$ means summable by Cesàro's $k$-th mean: see Proc. London Math. Soc., Ser. 2, Vol. 6, p. 257. By saying that $\Sigma a_{n}$ is finite $(C k)$, we mean that, in the notation of the paper just referred to, $S_{n}^{k} / A_{n}^{k}$ oscillates finitely as $n \rightarrow \infty$. 
are convergent, then the series $\Sigma a_{n} f_{n}$ is summable $(C k)$, and its sum is equal to that of the series

$$
\Sigma S_{n}^{k} \Delta^{k+1} f_{n}, *
$$

which is absolutely convergent.

This theorem is substantially the same as one which I proved in a paper † which I communicated to this Society in 1907, and which M. Bohr had not seen at the time of the publication of his note. It includes, as my theorem did not, the case in which $\Sigma a_{n}$ is only finite $(\mathrm{Ck})$. On the other hand the conditions are less simple than they may be made, in that [as Dr. Bromwich (Math. Annalen, Bd.65, p. 361) has proved] the convergence of $\Sigma n^{k}\left|\Delta^{k+1} f_{n}\right|$ involves that of all the other series mentioned in the final condition.

* The notation is that of the paper referred to in the preceding footnote.

+ Referred to in the two preceding footnotes.

[This paper unfortunately contains several inaccuracies, which have been pointed out to me by M. Bohr himself (see Bohr, Bidrag til de Diriclulet'ske Rackkers Theorie. Copenhagen, 1910) and by Mr. J. E. Littlewood. The fact is that, finding the whole difficulty of the investigation to lie in the algebraical work (pp. 258-261), I was careless in writing pp. 261-3, which do not involve any point of very serious difficulty.

In the first place, it should have been explicitly stated that, throughout the proof of Theorem I, the condition $f_{n} \rightarrow 0$ is assumed to be satisfied: this is, of course, implied in calling $f_{n}$ a " convergence factor" (p. 264). $§ \S 8,9,10$ should be modified as follows. Wc consider first the terms in $T_{11}^{k}$ for which $i=0$. These give

$$
{ }_{0} T_{n}^{k}=\sum_{j=0}^{n}\left(\begin{array}{c}
n-j+k \\
k
\end{array}\right) S_{j}^{k} \Delta^{k+1} f_{j} .
$$

In this expression $f_{m}$ is to be replaced by zero when $m>n$. But we may imagine this convention abandoned without affecting the limit of ${ }_{10} I_{n}^{k} / A_{n}^{k}$. For this introduces a set of new terms in number depending only on $k$; for these terms we have $n-j<K$, and so

$$
\left(\begin{array}{c}
n-j+k \\
k
\end{array}\right)<K
$$

and also

$$
\left|S_{j}^{k}\right| A_{n}^{k} \mid<K
$$

And, as each of them involves a factor $f_{m}$ which tends to zero as $n \rightarrow \infty$, their sum also tends to zero as $n \rightarrow \infty$.

Again,

$$
\left(\begin{array}{c}
n-j+k \\
k
\end{array}\right)-A_{n}^{k}=\frac{1}{k !}\{(n-j+1)(n-j+2) \ldots(n-j+k)-(n+1)(n+2) \ldots(n+k)\}
$$

is negative and numerically less than $K n^{k}(0 \leqslant j \leqslant n)$. Also since

$$
(n-j+1) \ldots(n-j+k)-(n+1)(n+2) \ldots(n+k)=-j \frac{d}{d x}\{(x+1)(x+2) \ldots(x+k)\}
$$


From this theorem M. Bohr deduces the existence of a line of summability $(\mathrm{Ck})$ for the series (2), viz.,

$$
R(s)=s_{k}=\varlimsup_{n \rightarrow \infty}\left\{\left(S_{n}^{k} k ! n^{-k}\right) / \log n\right\} .
$$

The series is summable $(C k)$ if $R(s)>s_{l}$, and not so summable if $R(s)<s_{k}$. Also

$$
0 \leqslant s_{k}-s_{k+1} \leqslant 1 \text {, }
$$

and the function represented by the series is regular for $R(s)>s_{k}$. Thus for the series (4), $s_{k}=-k$, and the series is summable, by one or other of Cesàro's means, all over the plane.

For the general series (1) there are wider possibilities as to summability. Thus, if $\lambda_{n}=n, s_{k}=s_{0}=\bar{s}$ for all values of $k$. On the other hand, the series

$$
\Sigma(-1)^{n-1} n^{\alpha}(\log n)^{-8} \quad(k-1<\alpha<k)
$$

where $n-j<x<n$, it follows that $\left(\begin{array}{c}n-j+k \\ k\end{array}\right)-A_{n}^{k}$ is numerically less than

$$
K \boldsymbol{\nu} n^{k-1} \quad(0 \leqslant j \leqslant \nu<n) .
$$

Hence

$$
\frac{{ }_{0} T_{n}^{k}}{A_{n}^{k}}=\epsilon_{n}+\sum_{0}^{n} S_{j}^{k} \Delta^{k+1} f_{j}+R_{0},
$$

where

$$
\left|R_{0}\right|<\frac{K_{\nu}}{n} \sum_{j=0}^{\nu} j^{k}\left|\Delta^{k+1} f\right|+K \sum_{\nu+1}^{n} j^{k}\left|\Delta^{k+1} f_{j}\right|:
$$

and by choosing first $\nu$ and then $n$ sufficiently large we see that $R_{0} \rightarrow 0$.

It follows that

$$
{ }_{0} T_{n}^{k} / A_{n}^{k} \rightarrow \sum_{0}^{\infty} S_{j}^{k} \Delta^{k+1} f_{j}
$$

Next we consider

$$
{ }_{i} T_{n}^{k}=\left(\begin{array}{c}
k+1 \\
i
\end{array}\right) \sum_{j=0}^{n}\left(\begin{array}{c}
n-j-i+k \\
k-i
\end{array}\right) S_{j}^{k} \Delta^{k+1-i} f_{j+i}
$$

Since

$$
\left(\begin{array}{c}
n-j-i+k \\
k-i
\end{array}\right)=(n-j+1)(n-j+2) \ldots(n-j+k-i) /(k-i) !<K n^{k-i} \text {, }
$$

it follows that

$$
\begin{aligned}
\left|\begin{array}{c}
i T_{n}^{k} \\
A_{n}^{i}
\end{array}\right| & <\sum_{n^{i}}^{K} \sum_{j=1}^{n}\left|S_{j}^{k} \Delta^{k+1-i} f_{j+i}\right|<\frac{K}{n^{i}} \sum_{j=0}^{n} j^{k}\left|\Delta^{k+1-i} f_{j+i}\right| \\
& <K \sum_{j=0}^{n}\left(\frac{j}{n}\right)^{i} j^{k-i}\left|\Delta^{k+1-i} f_{j+i}\right| .
\end{aligned}
$$

By dividing the range of summation into the two parts $(0, v),(\nu+1, n)$, and choosing first $\nu$ and then $n$ sufficiently large, we see that

$$
T_{n}^{k} / A_{n}^{k} \rightarrow 0 .
$$

This completes the proof of Theorem A, the statement of which requires no correction. The theorem, as $M$. Bohr points out, remains true when $f_{n} \rightarrow L(L \neq 0)$, provided that the expression for the sum of the series is modified by the addition of a term $S L$, where $S$ is the sum of the series $\Sigma a_{n}$. This follows at once from Theorem A on replacing $f_{n}$ by $f_{n}-L$.

Theorem $\mathrm{B}$ requires modification only in the replacing of $\Sigma S_{j}^{k} \Delta^{k+1} f_{j}$ by $\Sigma S_{j}^{\prime k} \Delta^{k+1} f_{j}$, wherc 
is summable $(C k)$ all over the plane, but never summable $(C, k-1)$, so that $s_{k}=-\infty, s_{k-1}=\infty$ : the sum represents an integral function of $s$.

3. I propose now to consider the application to the ordinary Dirichlet's series of Borel's method of summation. It is easy to see, by the consideration of two simple examples, that it either may or may not be possible, by the use of Borel's method, to continue a function partially represented by a Dirichlet's series outside the region of convergence of the series.

Suppose first that the series is $\Sigma n^{-s}$, which converges for $R(s)>1$, and represents the function $\zeta(s)$, whose only singularity is a simple pole for $s=1$. Then Borel's integral is

$$
\int_{0}^{\infty} e^{-x} u(x) d x
$$

$S^{k}$ is defined as in the text. Then $\left|S_{, j}^{\prime k}\right|<\epsilon A^{k}$ for $j \geqslant m_{0}$, and so

$$
\left|\sum_{m}^{m^{\prime}} S_{j}^{\prime k} \Delta^{k+1} f_{j}\right|<\epsilon \sum_{m}^{m^{\prime}} A_{j}^{k}\left|\Delta^{k+1} f_{j}\right|<\epsilon K,
$$

for $m^{\prime}>m \geqslant m_{0}$; whence the trath of the theorem follows.

The corollaries remain valid : but the first of them requires a few words of proof. The sum of the series $a_{0}^{\prime} f_{0}+a_{1}^{\prime} f_{1}+\ldots$ is $\Sigma S_{j}^{\prime k} \Delta^{k+1} f_{j}$, and is therefore continuous; and the sum of $a_{0} f_{0}+a_{1} f_{1}+\ldots$ differs from this by $S f_{0}$, and is therefore also continuous.

It is to be observed that the series

$$
\leq S_{j}^{k} \Delta^{k+1} f_{j}
$$

is, in general, neither uniformly convergent nor continuous. Suppose, e.g., that

$$
a_{0}=1, a_{1}=-1, a_{2}=1, \ldots,
$$

so that $k=1, S=\frac{1}{2}, a_{0}^{\prime}=1-\frac{1}{2}=\frac{1}{2}$; and that $f_{n}$ is a function, such as $x^{n}$, that has the limit 0 for $0 \leqslant x<1$, and the limit, 1 for $x=1$, the interval of values of $x$ under consideration being $0 \leqslant x \leqslant 1$. Then for $x<1$, we have

$$
\Sigma a_{n} f_{n}=\Sigma S_{j}^{1} \Delta^{2} f_{j}=\frac{1}{2} \Sigma(j+1) \Delta^{2} f_{j}+\Sigma S_{j}^{\prime \prime} \Delta^{z} f_{j}=\frac{1}{2} f_{0}+\Sigma S_{j}^{\prime} \Delta^{2} f_{j} ;
$$

the last series being a uniformly convergent series whose limit as $x \rightarrow 1$ is plainly zero. Thus $\Sigma a_{n} f_{n}$ and $\Sigma S_{j}^{1} \Delta^{2} f_{j}$ have the limit $\frac{1}{2}$. But the last series is discontinuous. For $x=1$ its sum is zero, but then

$$
\Sigma a_{n} f_{n}=L S+\Sigma S_{j}^{1} \Delta^{2} f_{j}=\frac{2}{2} .
$$

These facts, I may point out, are clearly recognised in my earlier paper in Vol. 2, Ser. 2, of these Proceedings (pp. 247 et seq.).

The genesis of the inaccuracies that $I$ have explained is to be found in a momentary confusion, more natural than excusable, between the relations

$$
S_{n}^{k} / A_{n}^{k} \rightarrow S, \quad S_{n}^{k} \rightarrow S .
$$

It is remarkable that so careless a blunder should not have led to more serious orror in my results.-Added, l'ebruary, 1910.] 
where*

$$
u(x)=\sum_{1}^{\infty} \frac{x^{n-1}}{n^{s}(n-1) !} .
$$

Since $\dagger$

$$
u(x) \sim x^{-s} e^{x},
$$

it follows that (6) is convergent (and then absolutely) if, and only if, $R(s)>1$, when the original series is convergent.

Secondly, consider the series (4), which represents an integral function of $s$. Here $\ddagger$

$$
u(x)=\sum_{1}^{\infty} \frac{(-x)^{n-1}}{n^{s}(n-1) !} \sim-\frac{1}{\Gamma(s)} x^{-1}(\log x)^{s-1} .
$$

Hence (6) is absolutely convergent for all values of $s$. It is easy to see that the same is true of

$$
\int_{0}^{\infty} e^{-x} u^{(\lambda)}(x) d x
$$

and so the series is absolutely summable all over the plane. $\$$

4. I shall now prove that, if the series $\Sigma a_{n}$ is summable, so is $\Sigma a_{n} n^{-s}$, where $R(s)>0$.

We are given that the integral (6) is convergent when

$$
u(x)=\sum_{1}^{\infty} \frac{a_{n} x^{n-1}}{(n-1) !} .
$$

It follows that the integral

$$
\int_{0}^{\infty} e^{-x} u(x y) d x
$$

is uniformly convergent for $0 \leqslant y \leqslant 1$. We have therefore, if $R(s)>0$, (9) $\int_{0}^{1}\left\{\log \left(\frac{1}{y}\right)\right\}^{s-1} d y \int_{0}^{\infty} e^{-x} u(x y) d x=\int_{0}^{\infty} e^{-x} d x \int_{0}^{1}\left\{\log \left(\frac{1}{y}\right)\right\}^{s-1} u(x y) d y$.

* We are applying Borel's method to $a_{1}+a_{2}+a_{3}+\ldots$ and not to $0+a_{1}+a_{2}+\ldots$. The equation would in the latter case be

$$
u(x)=\sum_{1}^{\infty} \frac{x^{n}}{n^{\frac{3}{3}} n !}
$$

The summability of $a_{1}+a_{3}+\ldots$ implies that of $0+a_{1}+a_{2}+\ldots$, wheress the converse is not true: see Bromwich, Infinite Series, p. 273, and a paper by the present writer in the Quarterly Journal, there referred to.

$\dagger$ Proc. London Math. Soc., Ser. 2, Vol. 2, p. 402.

$\ddagger$ Ibid. The formula fails if $s$ is zero or a negative integer; then $u(x)$ tends exponentially to zero as $x \rightarrow \infty$.

$\S$ Borel, Leçons sur les séries divergentes, p. 99.

|| Bromwich, Infinite Series, pp. 433 et seq. 
This follows from the following general theorem, which is in substance due to De lil Vallée-Poussin.* He considers only the cuse in which the variables $x, y$ are both real (which is all that is wanted here, the contour $C$ being the line $0 \leqslant y \leqslant 1$ ). We shall require the more general form of the theorem in $\$ 9$.

If $f(x, y)$ is a continuous function of the real variable $x$ and the real or cumplex variable $y$, and $\int_{0}^{\infty} f(x, y) d x$ is uniformly convergent for all values of $y$ lying on a finite contour $C$, and $\int_{C}|\phi(y)||d y|$ is convergent, then

$$
\int_{C} \phi(y) d y \int_{0}^{x} f(x, y) d x=\int_{0}^{\infty} d x \int_{C} \phi(y) f(x, y) d y .
$$

The following proof is an adaptation of the proof given by Dr. Bromwich (Infinite Series, p. 448) of an analogous theorem for series.

Since $\int_{0}^{\infty} f d x$ is a continuous function of $y$, the integral on the left hand is certainly convergent. Given $\epsilon$, we can choose $X$, so that $\left|\int_{x_{1}}^{x} f d x\right| \leqslant \epsilon$ for $x_{1} \geqslant X$. Also, in virtue of the continuity of $f$,

$$
\int_{C} \phi d y \int_{0}^{r_{1}} f d x=\int_{0}^{r_{1}} d x \int_{C} f \phi d y \text {. }
$$

Hence $\quad\left|\int_{C} \phi d y \int_{0}^{\infty} f d x-\int_{0}^{r_{1}} d x \int_{C} f \phi d y\right|=\left|\int_{C} \phi d y \int_{x_{1}}^{\infty} f d x\right| \leqslant \epsilon \int_{C}|\phi||d y|$,

for $x \geqslant X$, and so

$$
\begin{aligned}
& \int_{0}^{\infty} d x \int_{C} f \phi d y=\lim _{x_{1} \rightarrow \infty} \int_{11}^{x_{1}} d x \int_{C} f \phi d y=\int_{C} \phi d y \int_{0}^{\infty} f d x \\
& \text { Now } \int_{0}^{1}\left\{\log \left(\frac{1}{y}\right)\right\}^{s-1} u(x y) d y=\sum_{1}^{\infty} \frac{a_{u} x^{n-1}}{(n-1) !} \int_{u}^{1}\left\{\log \left(\frac{1}{y}\right) j^{s-1} y^{n-1} d y\right. \\
& =\Gamma(s) \sum_{1}^{\infty} \frac{a_{n} x^{n-1}}{n^{s}(n-1) !} \cdot \dagger
\end{aligned}
$$

'l'he last series represents the integral function associated with the series $\sum a_{n} n^{-8}$; and so this series is summable. Incidentally we have proved that its sum is

$$
\frac{1}{\Gamma(s)} \int_{0}^{1}\left(\log \left(\frac{1}{y}\right)\right)_{i}^{s-1} \psi(y) d y
$$

where $\psi(y)$ is the sum of the series $\Sigma a_{n} y^{n}$.

We notice further that the sum of the series $\Sigma a_{n} n^{-s}$ represents a function of $s$ regular for all values of $s$ whose real part is positive. This follows $\ddagger$ at once from the fact that

$$
\int_{0}^{1} \frac{\partial}{\partial s}\left\{\log \left(\frac{1}{y}\right)\right\}^{s-1} \psi(y) d y=\int_{0}^{1}\left\{\log \left(\frac{1}{y}\right)_{i}^{!^{s-1}} \log \log \left(\frac{1}{y}\right) \psi(y) d y\right.
$$

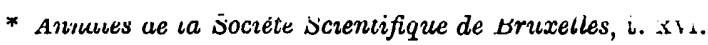

$\dagger$ The justification of the term-by-term integration presents no difficulty.

Bromwich, Infinite Series, p. 438. 
is uniformly convergent throughout any continuous region, in the plane of $s$, which lies entirely to the right of the imaginary axis.

We have thus established the existence of a line $R(s)=\sigma_{0}$, such that a Dirichlet's series is summable everywhere on its right and nowhere on its left. In other words, the region of summability is a half plane: it may, of course, include the whole plane $\left(\sigma_{0}=-\infty\right)$, or none of it $\left(\sigma_{0}=\infty\right)$.

5. I shall now prove further that, if the series $\Sigma a_{n}$ is absolutely summable, so is $\Sigma a_{n} n^{-8}$, where $R(s)>0$ : i.e., that the region of absolute summability is also a half plane.

We are assuming now that

$$
\int_{0}^{\infty} e^{-x}\left|u^{(\lambda)}(x)\right| d x
$$

is convergent for $\lambda=0,1,2, \ldots$, and we have to show that

$$
\int_{0}^{\infty} e^{-x}\left|u_{\mathrm{a}}^{(\lambda)}(x)\right| d x
$$

where

$$
u_{s}(x)=\sum_{1}^{\infty} \frac{a_{n} x^{n-1}}{n^{s}(n-1) !},
$$

is convergent. Now

$$
\begin{gathered}
u_{s}(x)=\frac{1}{\Gamma(s)} \int_{0}^{1}\left\{\log \left(\frac{1}{y}\right)\right\}^{s-1} u(x y) d y \\
\left|u_{s}(x)\right| \leqslant \frac{1}{|\Gamma(s)|} \int_{0}^{1}\left\{\log \left(\frac{1}{y}\right)\right\}^{R(s)-1}|u(x y)| d y .
\end{gathered}
$$

The integral

$$
\int_{0}^{\infty} e^{-x}|u(x y)| d x
$$

is uniformly convergent for $0 \leqslant y \leqslant 1$; and this enables us to show, by an argument precisely similar to that employed in the preceding section, that

$$
\int_{0}^{\infty} e^{-x} d x \int_{0}^{1}\left\{\log \left(\frac{1}{y}\right)\right\}^{R(s)-1}|u(x y)| d y,
$$

and, a fortiori,

is convergent. Again,

$$
\int_{0}^{\infty} e^{-x}\left|u_{s}(x)\right| d x
$$

$$
u_{s}^{(\lambda)}(x)=\frac{1}{\Gamma(s)} \int_{0}^{1}\left\{\log \left(\frac{1}{y}\right)\right\}^{s-1} u^{(\lambda)}(x y) y^{\lambda} d y,
$$


and, using this equation in exactly the same way, we can show that the remainder of the integrals (11) are convergent.

We thus establish the existence of a line of absolute summability

$$
R(s)=\bar{\sigma}:
$$

and clearly

$$
\sigma_{0} \leqslant \bar{\sigma}
$$

6. It is evident that the same reasoning might have been applied to the more general series

$$
\Sigma a_{n}\{\phi(n)\}^{-s},
$$

provided only that we could find an expression of $\{\phi(n)\}^{-8}$ in the form

$$
\{\phi(n)\}^{-s}=\int_{0}^{\infty} e^{-n v} \psi_{s}(w) d w=\int_{0}^{1} \psi_{s}\left\{\log \left(\frac{1}{y}\right) y_{j} y^{n-1} d y,\right.
$$

where $\psi_{s}$ is a function such that

is convergent.

$$
\left.\int_{0}^{1} \mid \psi_{s} \biguplus_{i} \log \left(\frac{1}{y}\right)\right\}_{i} \mid d y
$$

Suppose, for example, that

$$
\phi(n)=\theta(n+a)=(n+a)^{a_{0}}\{\log (n+a)\}^{a_{1}}\left\{\log _{2}(n+a)\right\}^{a_{2}} \ldots,
$$

the number of factors being finite, $\log _{2} n, \log _{3} n, \ldots$, denoting $\log \log n$, $\log \log \log n, \ldots$, and $a, a_{0}, a_{1}, \ldots$ being real, and $a$ and $a_{0}$ positive.

Then it may be deduced from a formula given by Pincherle that

$$
\{\theta(k)\}^{-s}=\int_{0}^{\infty} e^{-k w} \psi_{s}(w) d w
$$

where

$$
\psi_{s}(w)=\frac{1}{2 \pi i} \int_{\lambda-i \infty}^{\lambda+i \omega} e^{-v t}\{\theta(-t)\}^{-s} d t
$$

the path of integration being a straight line, and $e_{p-1}<-\lambda<k$, where $p$ is the number of logarithmic factors of $\theta(k)$, and $e_{0}, e_{1}, e_{2}, e_{3}, \ldots$ denote the numbers $1, e, e^{e}, e^{e^{e}}, \ldots$.

Hence we deduce

$$
\{\phi(n)\}^{-s}=\int_{0}^{\infty} e^{-(n+a) w} \psi_{s}(w) d w
$$

* Mem. di Bologna (4), t. 8, pp. 125 el seq. 
for all values of $n$ greater than a definite $n_{0}$. It may further be proved that as $w \rightarrow 0$,

$$
\psi_{s}(w) \sim \frac{w^{a_{s} s-1}}{\Gamma\left(a_{0} s\right)}\left\{\log \left(\frac{1}{w}\right)\right\}^{-a_{1} s} \ldots ;
$$

and, using these facts, we are able to extend the conclusions of $\S \S 4,5$ to the more general series

$$
\Sigma \frac{a_{n}}{(n+a)^{a_{n} s}\{\log (n+a)\}^{a_{1} s} \ldots} .
$$

On the other hand, it is by no means true that the regions of summability or of absolute summability of the general series (1) are necessarily half planes. It is sufficient to consider the series

$$
\sum_{1}^{\infty}(-1)^{n-1} e^{-n s}=\sum_{1}^{\infty}(-1)^{n-1} x^{n}=\frac{x}{1+x} .
$$

This series is summable (absolutely) if $R(x)>-1$. If $s=\xi+i \eta$, this condition is

$$
e^{-\xi} \cos \eta>-1
$$

and the region of summability is obtained by cutting out of the plane of $s$ an infinite succession of curvilinear areas whose general shape is easily sketched.

7. The series (4) is a simple example of a series summable by Borel's method all over the plane. For it Borel's method is more effective than any of Cesàro's : later on I shall define a large class of series, all of which resemble (4) in this respect. But it must not be imagined that Borel's method, even as applied to ordinary Dirichlet's series, is always as effective as even the simplest of Cesàro's; or that, even when the function represented by the series is regular all over the plane, it can always be continued by exponential summation.

Consider, for example, the series

$$
1^{-s}+0+0+\ldots-8^{-s}+0+\ldots+27^{-s}+0+\ldots,
$$

in which $a_{n}=(-1)^{k-1}$, when $n=k^{3}$, and $a_{n}=0$ otherwise. I. shall prove that this series is summable when, and only when, it is convergent, absolutely summable when, and only when, it is absolutely convergent. Thus the function

$$
\left(1-2^{1-3 s}\right) \xi(3 s)
$$


represented by the series when $R(s)>0$, although an integral function of $s$, cannot be continued by the use of Borel's method.

It is convenient to consider instead of (12) the series

$$
0+1^{-s}+0+\ldots-8^{-s}+0+\ldots+27^{-s}+\ldots,
$$

which is certainly summable (absolutely summable) if (12) is summable (absolutely summable). Then

$$
u(x)=\sum_{1}^{\infty} \frac{(-1)^{n-1}}{n^{3 s}} \frac{x^{n !}}{\left(n^{3}\right) !} .
$$

I have considered elsewhere* the asymptotic properties of functions of this type, but it will be necessary now to obtain rather more precise information.

We divide the range of integration into two sets of intervals $i_{v}, j_{v}$, $i_{\nu}$ being the interval $(\nu-\delta)^{3} \leqslant x \leqslant(\nu+\delta)^{3}$, where $\delta$ is a small fixed positive number. We shall consider first whether the series

is convergent.

$$
\sum_{(v)} \int_{\left(i_{v}\right)} e^{-x} u(x) d x
$$

If

$$
v_{n}=n^{-3 s} x^{n^{*}} /\left(n^{3}\right) !,
$$

we may write $u(x)$ in the form

$$
\begin{aligned}
u(x) & =(-1)^{\nu-1} v_{\nu}+\left(\sum_{1}^{\nu-1}+\sum_{\nu+1}^{D}\right)(-1)^{n-1} v_{n} \\
& =(-1)^{\nu-1} v_{\nu}+S_{1}+S_{\nu},
\end{aligned}
$$

say. It is easy to see that, when $x$ lies in $i_{v}, v_{v}$ is, to a first approximation, of the order of $e^{x}$ : a more precise approximation will be obtained later. A straightforward application of Stirling's theorem gives the formula

$$
v_{n} / v_{n+1}=\left(1+\epsilon_{n}\right) x^{-3 n^{2}-3 n-1} \exp \left\{\left(9 n^{2}+9 n+3\right) \log n+\frac{9}{2}(n+1)\right\},
$$

where $\epsilon_{n} \rightarrow 0$ as $n \rightarrow \infty$. If $n \geqslant \nu$, we have

$$
\begin{aligned}
x^{-3 n^{2}-3 n-1} & >(\nu+\delta)^{-9 n^{2}-9 n-3} \geqslant(n+\delta)^{-9 n^{2}-9 n-3} \\
& >n^{-9 n^{2}-9 n-3} \exp \frac{1}{n}\left(9 n^{2}+9 n+3\right) ; \\
& >K n^{-9 n^{2}-9 n-3} e^{-9 \delta n},
\end{aligned}
$$

- Proc. London Math. Soc., Ser. 2, Vol. 2, pp. 335 et seq.; Messenger of Math., Vol. 39, pp. 28 et seq. 
and, accordingly,

$$
v_{n} / v_{n+1}>K e^{(i-\delta) n}>e^{4 n}:
$$

and it follows that

$$
\left|S_{2}\right|<K e^{-4 \nu}\left|v_{\nu}\right| \text {. }
$$

$$
\text { Again, } \quad S_{1}=\left(\sum_{1}^{n_{0}}+\sum_{n_{n}+1}^{\nu-1}\right)(-1)^{n-1} v_{n}=S_{1}^{\prime}+S_{1}^{\prime \prime} \text {, }
$$

say. We choose $n_{0}$, once for all, so as to justify the application of Stirling's theorem to the terms for which $n>n_{0}$. Then, if $n_{0}<n<\nu$, we have

$$
\begin{aligned}
x^{-9 n^{2}-3 n-1} & <(\nu-\delta)^{-9 n^{2}-9 n-3} \leqslant(n+1-\delta)^{-9 n^{2}-9 n-3} \\
& <n^{-9 n^{2}-9 n-3} \exp \left[-\left\{\frac{1-\delta}{n}-\frac{(1-\delta)^{2}}{2 n^{2}}\right\}\left(9 n^{2}+9 n+8\right)\right] \\
& <K n^{-9 n^{2}-9 n-3} e^{-9(1-\delta) n},
\end{aligned}
$$

and so

$$
v_{n} / v_{n+1}<K e^{-(1-\delta) n}<e^{-4 n} .
$$

It follows that

$$
\left|S_{1}^{\prime \prime}\right|<K e^{-4 v}\left|v_{v}\right| \text {. }
$$

Also $S_{1}^{\prime \prime}$ is a polynomial in $n$, of degree $n_{0}^{8}$, while $v_{\nu}$ is (roughly) of order $e^{x}$

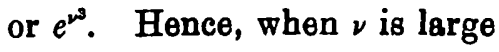

$$
\left|S_{1}^{\prime}\right|<K e^{-4 \nu}\left|v_{v}\right| \text {. }
$$

From (14), (15), and (16) it follows that, in $i_{v}$,

$$
u(x)=(-1)^{\nu-1} v_{\nu}\left(1+\rho_{\nu}\right),
$$

where

$$
\left|\rho_{\nu}\right|<K e^{-4 \nu} \text {. }
$$

Again, $\quad v_{\nu}=\frac{\nu^{-8 s} x^{\nu^{3}}}{\left(\nu^{8}\right) !}=\frac{\nu^{-3 s}(\nu+t)^{3 \nu^{s}}}{\left(\nu^{3}\right) !} \quad(|t|<\delta)$

$$
=\frac{\nu^{-9 s}}{\sqrt{ }(2 \pi)} \exp \left\{3 \nu^{3} \log (\nu+t)-3\left(\nu^{3}+\frac{1}{2}\right) \log \nu+\nu^{3}+r_{\nu}\right\},
$$

where

$$
\left|r_{v}\right|<K / \nu^{8} \text {. }
$$

We easily deduce that

$$
v_{\nu}=\frac{\nu^{-s s-t}}{\sqrt{ }(2 \pi)} e^{x-s v^{2}}\left(1+\epsilon_{\nu}\right),
$$

where

$$
\left|\epsilon_{\nu}\right|<K / \nu \text {. }
$$

From this it follows that

$$
\left|e^{-x}\left\{u(x)-(-1)^{\nu-1} v_{v}\right\}\right|<K \nu^{-8 R(s)-\frac{s}{s}} e^{-4 \nu},
$$

which is obviously, whatever the value of $s$, even when multiplied by the 
length of the interval $i_{\nu}$, the general term of an absolutely convergent series. Hence the convergence of (13) depends entirely upon that of the series

or of

$$
\Sigma(-1)^{\nu-1} \int_{\left(i^{\nu}\right)} e^{-v} v_{v} d x
$$

$$
\Sigma(-1)^{\nu-1} \nu^{-3 s-1} \int_{(\nu-\delta)^{3}}^{(\nu+\delta)^{3}} e^{-\frac{8}{3} \nu^{2}}\left(1+\epsilon_{\nu}\right) d x .
$$

Now

$$
\begin{aligned}
& \int_{(\nu-\delta)^{4}}^{(\nu+\delta)^{3}} e^{-\frac{8}{5} \nu t^{2}} d x=\mathbf{3} \int_{-\delta}^{\delta}(\nu+t)^{2} e^{-\frac{8}{2} \nu \nu^{2}} d t \\
& =6 \int_{0}^{\delta}\left(\nu^{2}+t^{2}\right) e^{-s \nu v^{2}} d t \\
& =6 \nu^{2}\left(1+\eta_{v}\right) \int_{0}^{\infty} e^{-\frac{\beta}{v} v^{2}} d t \\
& =\nu^{*} \sqrt{ }(2 \pi)\left(1+\eta_{\nu}\right),
\end{aligned}
$$

where

$$
\left|\eta_{\nu}\right|<K / \nu_{0}^{*}
$$

Hence the series (19) may be written in the form

$$
\sqrt{ }(2 \pi) \Sigma(-1)^{\nu-1} \nu^{-3 s}\left(1+\eta_{\nu}\right) ;
$$

and this series is absolutely convergent if $R(s)>\frac{1}{3}$, and conditionally convergent if $0<R(s) \leqslant \frac{1}{3}$.

It remains to consider the series

$$
\Sigma \int_{\left(j_{v}\right)} e^{-x} u(x) d x
$$

where $j_{\nu}$ is the interval $(\nu+\delta)^{3} \leqslant x \leqslant(\nu+1-\delta)^{3}$. But it follows at once from the work in my note in the Messenger of Mathematics quoted above that, in $j_{v}$,

$$
\left|e^{-x} u(x)\right|<K_{1} x^{K_{2} R(s)+K_{3}} e^{-K_{4} x^{x}},
$$

where $K_{1}, K_{2}, K_{3}$ and $K_{4}$ are constants : and from this it follows that the series (22) is absolutely convergent for all values of $s$. And this completes the proof of the assertion made at the beginning of this section.

The same conclusions may be extended to the series

$$
\left(1^{k}\right)^{-s}+0+\ldots-\left(2^{k}\right)^{-s}+0+\ldots+\left(3^{k}\right)^{-s}+0+\ldots,
$$

where $l_{i}$ is any integer greater than 2.

* A smaller limit may be assigned for $\left|\eta_{v}\right|$, but this one is sufficient for our present purpose. SER. 2. VoL. 8. No. 1054 . 
8. The series considered in the last section are interesting as examples of the possibilility of Borel's method proving less powerful than Cesàro's. For it follows from investigations of Cesirro and myself* that the summability $(C 1)$ of the series

$$
a_{1}+a_{2}+a_{3}+\ldots
$$

involves that of all the series

$$
\begin{gathered}
a_{1}+0+0+a_{2}+0+0+0+0+a_{1}+0+\ldots, \\
a_{1}+0+0+0+0+0+0+a_{2}+0+0+0+0+\ldots,
\end{gathered}
$$

in which $a_{v}$ occur's respectively in the $\nu^{2}$-th, $\nu^{3}$-th, ... position. Hence, for example, the series (12) is summable $(C 1)$ for $-\frac{1}{3}<R(s) \leqslant 0$ : it may lie proved to be summable $(C k)$ for $-\frac{1}{3} k<R(s) \leqslant-\frac{1}{3}(k-1)$; and so summable by one or other of Cesàro's means all over the plane.

9. Let us call

$$
\Sigma a_{n} n^{-s}
$$

the Dirichlet's series associated with the power series

$$
\Sigma a_{n} x^{n}
$$

If (24) has a radius of convergence greater than unity, (23) is absolutely convergent for all values of $s$, and represents an integral function of $s$. If the radius of convergence of (24) is less than unity, (23) is never convergent. If the radius of convergence of (24) is unity, (23) may converge for none or for some or for all values of $s$; examples are given by the series

$$
\Sigma e^{v^{n}} n^{-s}, \quad \Sigma n^{-s}, \quad \Sigma c^{-\sqrt{ } n} n^{-s} \text {. }
$$

I shall assume only that the radius of convergence of (24) is positive: the series then possesses a circle of convergence and a polygon of summability.t And I shall now prove the following theorem :-

If the point $x=1$ lies within the polygon of summability of the series (24), the associated Dirichlet's series is summable by Borel's method for all values of $s$, and represents an integral function of $s$.

* See Quarterly Journal, Yol. 35, pp. 260 ct seq.: B3romwich, Infinite Series, pp. 386 et seq.

† Borcl, Leçons sur les séries divergentes, p. 126 ; Bromwich, Infinite Series, p. 295. 
Borel's sum is given by the integral

$$
\int_{0}^{\infty} e^{-x} u_{s}(x) d x
$$

where

$$
u_{s}(x)=\sum_{1}^{\infty} \frac{a_{n} x^{n-1}}{n^{8}(n-1) !} \text {. }
$$

Now

$$
n^{-s}=\frac{i \Gamma(1-s)}{2 \pi} \int_{W}(-w)^{s-1} e^{-u v} d w,^{*}
$$

where

$$
(-w)^{s-1}=\exp \{(s-1) \log (-v(1)\},
$$

the logarithm being real when $w$ is real and negative, and where $W$ represents a contour beginning and ending at the infinitely distant end of the positive real axis and surrounding the positive real axis by a counter-clockwise circuit. We shall suppose that the point of $W$, where $R(w)$ has its algebraical minimum, is its point of intersection with the negative real axis, where $w=-\delta, \delta$ being a positive number as small as we please.

The equation (27) holds for all values of $s$ save $s=1,2,3, \ldots$ : values which we shall at present omit from consideration.

From (27) we at once deduce

$$
\begin{aligned}
u_{s}(x) & =\frac{i \Gamma(1-s)}{2 \pi} \int_{W}(-v)^{s-1} e^{-w} u\left(x e^{-w}\right) d w \\
& =\frac{i \Gamma(1-s)}{2 \pi} \int_{T}(\log t)^{s-1} u(x t) d t,
\end{aligned}
$$

where $T$ is a loop from the origin in the plane of $T$ round the point $t=1$, as shown in the figure. The term by term integration here employed is of a type whose justification presents no difficulty.

We shall now prove that

$$
\int_{0}^{\infty} e^{-x} d x \int_{T}(\log t)^{s-1} u(x t) d t=\int_{T}(\log t)^{s-1} d t \int_{0}^{\infty} e^{-x} u(x t) d x ;
$$

or, in other words, that the integral (25) is convergent, and may be calculated by an inversion of the order of integration.

The integral

$$
\int_{0}^{\infty} e^{-x} u(x t) d x
$$


is uniformly (and absolutely) convergent throughout any region in the plane of $t$ which lies entirely inside the polygon of summability of the series $\Sigma a_{n} t^{n}$. If $t=1$ lies inside the polygon it is evident (see the figure) that we can draw $W$ so that $T$ lies entirely in the polygon, and

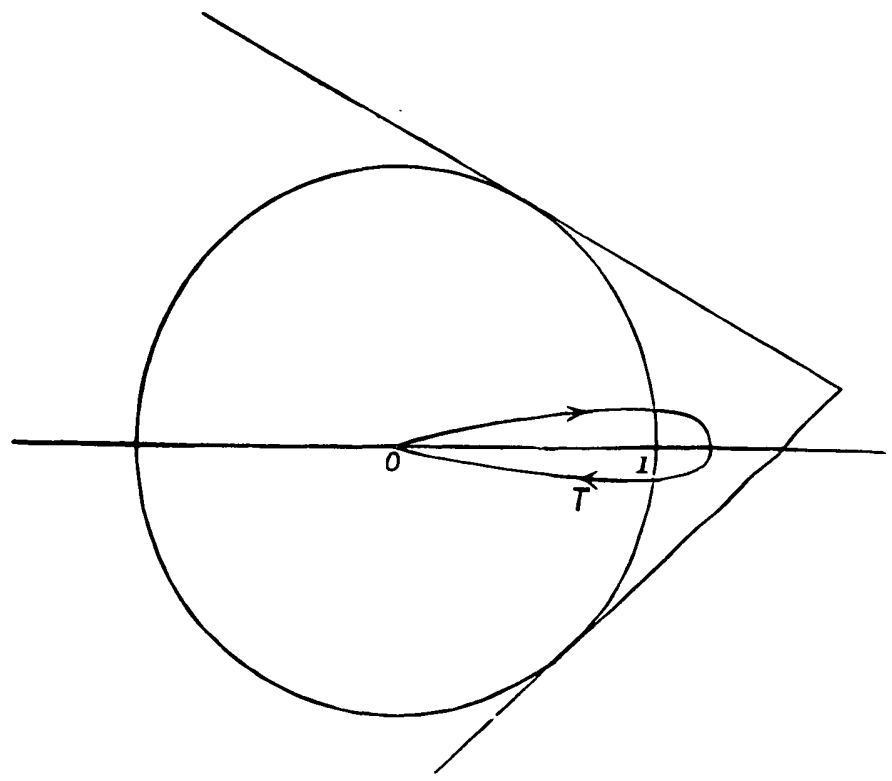

then (30) is uniformly convergent for all values of $t$ on $T$. Our conclusion then follows at once from the auxiliary theorem of $\S 4$.

The proof that the "sum" of the series represents an analytic function regular all over the plane may now be supplied as in $\$ 4$. Incidentally we see that the sum is

$$
\frac{i \Gamma(1-s)}{2 \pi} \int_{T}(\log t)^{s-1} \phi(t) d t,
$$

where $\phi(t)$ is the function represented by $\Sigma a_{n} t^{n}$ and its continuation by Borel's integral.

So far we have assumed that $s$ is not a positive integer: if it is we replace (27) by the equation

$$
n^{-s}=\frac{1}{\Gamma(s)} \int_{0}^{\infty} w^{s-1} e^{-n w} d w
$$

and argue in precisely the same way. 
10. The conclusions of the last section may be extended (cf. $\$ 6$ ) to more general series of the type $\Sigma a_{n}\{\phi(n)\}^{-s}$, such as

$$
\sum_{n_{0}}^{\infty} \frac{a_{n}}{(n+a)^{a_{0} s}\{\log (n+a)\}^{a_{1} s} \ldots} .
$$

It is to be observed that they are quite independent of any assumption that the Dirichlet's series is ever convergent: thus they apply to such series as

$$
\Sigma(-1)^{n-1} a^{n} n^{-}, \quad \Sigma(-1)^{n-1} a^{2 n+1}(2 n+1)^{-s},
$$

where $a$ is any positive number, however large. But certainly the most interesting case of the theorem is :

If $\Sigma a_{n} x^{n}$ is convergent for $|x|<1$, and the function represented by the series is regular for $x=1$, then the associated Dirichlet's series is summable for all values of $s$, and represents an integral function of $s$.

The last assertion (having no reference to summability) is easily established directly. As examples of series which satisfy these conditions, we may mention

$$
\begin{aligned}
& \Sigma \frac{\cos n \theta}{n^{s}}, \quad \Sigma \frac{\sin n \theta}{n^{s}}, \\
& \Sigma \frac{1}{n_{0}^{\beta}(\log n)^{\beta_{1}} \ldots} \frac{e^{n i \theta}}{n^{s}},
\end{aligned}
$$

where

$$
\theta \not 0 \quad(\bmod 2 \pi) \text {. }
$$

With the help of the remarks made at the beginning of this section the conclusion may be extended to such series as, e.g.,

$$
\Sigma \frac{\xi^{n} e^{n i \theta}}{(n+b)^{\beta_{0}}\{\log (n+b)\}^{\beta_{2}} \ldots\left[(n+a)^{\alpha_{0}}\{\log (n+a)\}^{\alpha_{1}} \ldots\right]^{s}} .
$$

11. It is instructive to verify the results of $\$ \S 9,10$ in the case of the simple series (31). Writing $2 \pi \phi$ for $\theta$, and using Hurwitz's formula*

$$
\xi(s, \phi)=2 \Gamma(1-s)(2 \pi)^{s-1} \sum_{1}^{\infty} n^{-(1-s)} \sin \left\{\left(2 n \phi+\frac{1}{2} s\right) \pi\right\},
$$

where

$$
0<\phi<1, \quad R(s)<1,
$$

and $\xi(s, \phi)$ is the generalised Riemann $\xi$-function defined by the series

$$
\phi^{-s}+(\phi+1)^{-s}+(\phi+2)^{-s}+\ldots
$$

- Lindelöf, Le calcul des résidus, p. 107. 
294 Application of Borel's exponential method of summation. [Nov. 11, and its continuations, we see that

$$
\begin{aligned}
& C_{s}^{\prime}(\phi)=\sum_{1}^{\infty} \frac{\cos 2 n \pi \phi}{n^{s}}=\frac{(2 \pi)^{1-s}}{4 \Gamma(1-s) \sin \frac{1}{2} s \pi}\{\xi(s, \phi)+\xi(s, 1-\phi)\}, \\
& S_{s}(\phi)=\sum_{1}^{\infty} \frac{\sin 2 n \pi \phi}{n^{s}}=\frac{(2 \pi)^{1-s}}{4 \Gamma(1-s) \cos \frac{1}{2} s \pi}\{\xi(s, \phi)-\xi(s, 1-\phi)\} .
\end{aligned}
$$

The functions $\xi(s, \phi), \xi(s, 1-\phi)$ are each regular save for a simple pole, with unit residue, at $s=1$. It is clear that the only possible singularities of $C$ and $S$ are simple poles at the points $s=0,-2,-4, \ldots$ and $s=-1,-3,-5, \ldots$ respectively. To verify that $C$ and $S$ axe in fact regular at these points, we have to show that

$$
\xi(-2 k, \phi)+\xi(-2 k, 1-\phi)=0, \quad \xi(-2 k-1, \phi)=\xi(-2 k-1,1-\phi):
$$

and these equations are easily verified by the help of known results in the theory of the Zeta and Bernoullian functions.*

12. If $\Sigma a_{n} x^{n}$ is regular for $|x|<1$, and has a simple pole for $x=1$, then $\sum a_{n} n^{-s}$ is regular all over the plane except for a simple pole at $s=1$. If $\Sigma a_{n} x^{n}$, at $x=1$, behaves like

$$
(1-x)^{a} \phi(x)
$$

where $\phi(x)$ is regular, then $\Sigma a_{n} n^{-s}$ has simple poles for

$$
s=-\alpha,-1-\alpha,-2-a, \ldots
$$

unless $a$ is integral. If $a$ is a positive integer, there are no poles; if a negative integer, the poles are

$$
1,2, \ldots,-\alpha .
$$

These results follow at once from the consideration of the equation

$$
\Sigma a_{n} n^{-s}=\frac{i}{2 \Gamma(s)\{\sin (\alpha+s) \pi\}} \int_{11}(-w)^{u+s-1} \chi\left(e^{-w}\right) d w,
$$

where $\chi\left(e^{-w}\right)$ is a function regular for $w=0$. I do not imagine that they are new : but they are worth stating here in connection with the results of $\$ \$ 9-11$.

- See Barnes, Messenger of Mathematics, Vol. xxix, pp. 74 et seq, 88 et seq. 